\title{
Efficacy and tolerability of chemotherapy with modified dose-dense TCF regimen (TCF-dd) in locally advanced or metastatic gastric cancer: final results of a phase II trial
}

\author{
Gianluca Tomasello $\cdot$ Wanda Liguigli $\cdot$ Rossana Poli $\cdot$ Silvia Lazzarelli • \\ Matteo Brighenti - Federica Negri - Alessandra Curti - Mario Martinotti • \\ Lucio Olivetti - Massimo Rovatti - Gianvito Donati $\cdot$ Rodolfo Passalacqua
}

Received: 22 May 2013/ Accepted: 10 November 2013/Published online: 27 November 2013

(C) The International Gastric Cancer Association and The Japanese Gastric Cancer Association 2013

\begin{abstract}
Background We previously studied a dose-dense TCF (TCF-dd) regimen demonstrating its feasibility and an activity comparable to epirubicin-based chemotherapy and TCF $\mathrm{q} 3 \mathrm{w}$ in terms of overall survival and time to progression (TTP). We report here the final results of a phase II study of chemotherapy with a modified TCF-dd regimen in locally advanced or metastatic gastric cancer (MGC).

Methods and study design Patients with histologically confirmed measurable MGC, not previously treated for advanced disease, received docetaxel $70 \mathrm{mg} / \mathrm{m}^{2}$ day 1 , cisplatin $60 \mathrm{mg} / \mathrm{m}^{2}$ day 1,1 -folinic acid $100 \mathrm{mg} / \mathrm{m}^{2}$ days 1 and 2 , followed by 5 -fluorouracil ( 5 -FU) $400 \mathrm{mg} / \mathrm{m}^{2}$ bolus days 1 and 2 , and then $600 \mathrm{mg} / \mathrm{m}^{2}$ as a 22-h continuous infusion days 1 and 2, every 14 days, plus pegfilgrastim $6 \mathrm{mg}$ on day 3 . Patients aged $\geq 65$ years received the same schedule with a dose reduction of $30 \%$.

Results Study duration: December 2007-November 2010. Forty-six consecutive patients were enrolled (78\% male, $22 \%$ female; median age, 66 years, range, 38-76 years; ECOG PS: 0, $48 \%, 1,46 \%$ ). Primary endpoint was overall response rate (ORR). A median of four cycles
\end{abstract}

G. Tomasello ( $₫)$ · W. Liguigli · R. Poli · S. Lazzarelli • M. Brighenti - F. Negri - A. Curti - G. Donati - R. Passalacqua Medical Oncology Division, Azienda Istituti Ospitalieri di Cremona, Viale Concordia 1, 26100 Cremona, Italy

e-mail: g.tomasello@ospedale.cremona.it

M. Martinotti · M. Rovatti

Surgery Division, Azienda Istituti Ospitalieri di Cremona,

Viale Concordia 1, 26100 Cremona, Italy

L. Olivetti

Radiology Division, Azienda Istituti Ospitalieri di Cremona,

Viale Concordia 1, 26100 Cremona, Italy (range, one to six) was administered. Forty-three patients were evaluated for response (93.5\%) and all for toxicity: 3 complete response (CR), 25 partial response (PR), 10 stable disease (SD), and 5 progressive disease (PD) were observed, for an ORR by intention to treat (ITT) of $61 \%$ (95\% CI 47-75). Median overall survival (OS) was 17.63 months (95\% CI, 13.67-20.67); median progression-free survival was 8.9 months $(95 \% \mathrm{CI}, 6.5-13.4)$. Twenty-one patients $(46.0 \%)$ were treated at full doses without any delay, thus respecting the dose-dense criterion. Most frequent grade 3-4 toxicities were neutropenia $(20 \%)$, leukopenia (4\%), thrombocytopenia (2\%), anemia $(2 \%)$, febrile neutropenia (6\%), asthenia (22\%), diarrhea (4\%), nausea/vomiting (11\%), and hypokalemia (6\%). Overall, TCF-dd was shown to be safe.

Conclusions The TCF-dd regimen in locally advanced or MGC is confirmed to be feasible and very active and needs to be further tested in randomized studies.

Keywords Gastric cancer - Chemotherapy - TCF . Dose-dense

\section{Introduction}

Background

Gastric cancer is the second leading cause of cancer death and the fourth most common type of cancer. Globally, 989,600 new cases and 738,000 deaths per year can be encountered [1].

Only $3.1 \%$ of patients with advanced gastric cancer survives up to 5 years, and the role of surgery is limited to $23 \%$ of patients, which often depends on the delayed diagnosis because of nonspecific symptoms [2]. 
The goal of chemotherapy in metastatic gastric cancer (MGC) is to improve quality of life.

In the 1990s, three randomized trials [3-5] evaluated the benefit of chemotherapy versus supportive care alone, showing clearly its beneficial effect. A significant improvement in overall survival (OS) in favor of chemotherapy was also confirmed in a subsequent metanalysis of phase II-III clinical trials [6].

Very few studies have compared single-agent chemotherapy (mainly represented by 5-FU) with combination regimens, showing better response rates but minimal clinically significant survival benefits for polychemotherapy [7-9].

Fluoropyrimidines plus platinum derivatives [10], epirubicin [11], and, more recently, taxanes [12, 13] and irinotecan [14] represent the most active drugs in the treatment of advanced disease.

Despite the availability of numerous effective drugs and different combinations, it is still not possible to define a recommended chemotherapy regimen in patients with gastric cancer that is HER2 negative or with unknown receptor status. From the data available so far, the use, in patients with a good performance status, of a three-drug combination chemotherapy seems feasible. Randomized phase II [15] and phase III studies [16], on triplets as ECF (epirubicin, cisplatin, and 5-FU) and TCF (docetaxel), have shown an increase in response rate from $35 \%$ to $40 \%$ with a median survival estimated between 8 and 11 months. However, data on toxicity despite the clinical benefit [17] and an improved quality of life [18] highlight the need to move toward new schedules of administration.

We previously tested at our center, in a pilot study conducted in a different and separate group of patients (enrolled from November 2004 until June 2007), the feasibility and effectiveness of a TCF regimen modified according to a dose-dense schedule [19].

Some randomized clinical trials have already shown the potential benefits of dose-dense chemotherapy in neoplasms such as breast [20], non-Hodgkin lymphoma [21], and bladder cancer [22]. The availability of granulocyte growth factors has permitted reducing the incidence of neutropenia, allowing us to take advantage of the Norton-Simon hypothesis [23], according to which efficacy of chemotherapy increases by reducing the interval between treatment cycles.

The first studies on dose-density in gastric cancer date back to the late 1980s when regimens containing metrothexate, 5-FU, and doxorubicin (FAMTX) and then 5-FU, cisplatin, and epirubicin (PELF) were tested [24].

TCF q3w certainly represents one of the most effective regimes and is a reference three-drug regimen worldwide used in MGC, which is generally a chemosensitive disease.

To further improve its performance in terms of response rate maintaining an acceptable toxicity profile, we designed an innovative polychemotherapy scheme. Here we report the final efficacy and toxicity results of a phase II study with this modified intensified dose-dense TCF regimen.

\section{Methods}

Trial design

This was a nonrandomized, open-label, single-center phase II study of dose-dense chemotherapy with modified TCF regimen in locally advanced or MGC.

\section{Participants}

Main entry criteria of the study included histologically or cytologically confirmed gastric cancer, locally advanced nonresectable primary tumor, presence of measurable or evaluable tumor lesions, age $\geq 18$ years, and adequate hepatic, renal, bone marrow, and cardiac function. Prior adjuvant chemotherapy and radiotherapy were allowed provided that these interventions had been completed at least 6 months before enrollment in the study. Major exclusion criteria were an ECOG PS $\geq 2$, prior palliative chemotherapy, pregnancy, breast-feeding, child-bearing potentiality without use of any contraception, any other current or prior malignancy (with the exception of excised cervical carcinoma in situ or squamous cell skin carcinoma), and psychiatric disorders potentially affecting compliance to the therapeutic program. All patients provided written informed consent. The trial protocol was approved by the local ethics committee.

\section{Interventions}

Upon study entry, a complete medical history was taken, and all the patients underwent a physical examination, evaluation of ECOG PS, blood chemistry tests, computed tomography scan of the abdomen, of the chest, and of all measurable and assessable sites. Bone scan, magnetic resonance imaging scan, and ultrasound endoscopy were carried out only if clinically indicated. Patients subsequently underwent a physical examination and laboratory tests (blood cell count, serum creatinine, bilirubin, AST, ALT) before each cycle of treatment. Tumor evaluations were carried out every 2 months until disease progression or withdrawal from study medication, on the basis of the response evaluation criteria in solid tumors (RECIST) criteria version 1.0. In addition, survival was monitored every 2 months in each patient leaving the study. Adverse events were classified according to National Cancer Institute (NCI) common toxicity criteria (CTC), version 3.0.

The TCF-dd regimen consisted of docetaxel (Taxotere; Sanofi-Aventis, Paris, France), $70 \mathrm{mg} / \mathrm{m}^{2}$ over a 1-h 
intravenous (i.v.) infusion on day 1 ; cisplatin, $60 \mathrm{mg} / \mathrm{m}^{2}$ on day 1 (1- to 3-h i.v. infusion); l-folinic acid, $100 \mathrm{mg} / \mathrm{m}^{2}$ administered in $5 \%$ glucose over $2 \mathrm{~h}$ i.v. on days 1 and 2; followed by 5 -FU, $400 \mathrm{mg} / \mathrm{m}^{2}$ bolus i.v. on days 1 and 2 , and then 5 -FU, $600 \mathrm{mg} / \mathrm{m}^{2}$ as a continuous i.v. infusion over $22 \mathrm{~h}$ on days 1 and 2. Pegfilgrastim (Neulasta; Amgen, Thousand Oaks, CA, USA), $6 \mathrm{mg}$, was administered subcutaneously on day 3 . Patients aged $\geq 65$ years received the same schedule with a dose reduction of all agents by $30 \%$.

Anti-emesis treatment (5-hydroxytryptamine receptor antagonists and dexamethasone), appropriate hydration, and corticosteroid premedications were always administered before chemotherapy infusion. Treatment was repeated every 14 days and was continued for up to six cycles (one cycle $=14$ days) in the absence of disease progression, unacceptable toxicity, patient's refusal, or physician's decision. Treatment was delayed in case of insufficient hematological function (neutrophil count $<1,500 / \mathrm{mm}^{3}$ and/ or platelet count $<100,000 / \mathrm{mm}^{3}$ ), and/or nonhematological toxicity grade $>1$ on day 15 of any cycle. If toxicity lasted longer than 2 weeks, the treatment was continued, after recovery, with a dose reduction by $20 \%$, but always maintaining the 2-week schedule. In the event of febrile neutropenia, grade 4 nonfebrile neutropenia lasting longer than 5 days, or grade 4 or grade 3 with bleeding thrombocytopenia, the dose of each drug was reduced by $25 \%$. The same dose reduction was indicated for grade 3 and 4 nonhematological toxicity.

\section{Objectives of the study}

The primary objective was the activity evaluated as ORR (complete + partial responses). Secondary endpoints were PFS, toxicity, and OS.

Sample size and statistical methods

To measure the real advantage of the biweekly scheme, we assumed a $20 \%$ improvement in response rate compared to reported data in the literature (classical TCF: ORR $37 \%$ ). According to Simon's two-stage design, setting a $5 \%$ alpha significance level and an $80 \%$ power of the study, the estimated total number of patients to be enrolled was 46. The first stage involved the recruitment of 15 patients. If the number of observed responses would have been $<5$, the recruitment would be stopped and the experimental treatment considered equal to the reference treatment in terms of response. If the number of responses observed would have been $>5$, then 31 additional patients would have been enrolled. The experimental treatment had to be considered effective if the total number of responses observed were $>18$.
The efficacy analyses were based on the intent-to-treat population. Descriptive statistics were reported as proportions and medians. Kaplan-Meier estimates were used in the analysis of time-to-event variables, and the $95 \%$ confidence interval $(\mathrm{CI})$ for the median time to event was computed.

Patients who have not received at least one dose of the drug were excluded from the analysis of safety.

\section{Results}

Patients' characteristics

Forty-six patients were enrolled from December 2007 to November 2010. Patient characteristics are reported in Table 1. Median age was 66 years (range, 38-76); $78 \%$ of patients were male and $22 \%$ female. Twenty-two patients had an ECOG PS of $0(48 \%), 21$ had a PS of $1(46 \%)$, and

Table 1 Patient characteristics

\begin{tabular}{|c|c|c|}
\hline & $n=46$ & $\%$ \\
\hline \multicolumn{3}{|l|}{ Sex } \\
\hline Male & 36 & 78 \\
\hline Female & 10 & 22 \\
\hline Age (year), median (range) & $66(38-76)$ & \\
\hline ECOG PS 0 & 22 & 48 \\
\hline 1 & 21 & 46 \\
\hline 2 & 3 & 6 \\
\hline \multicolumn{3}{|l|}{ Histological diagnosis } \\
\hline Adenocarcinoma G: unknown & 6 & 13 \\
\hline G1 & 1 & 2 \\
\hline G2 & 12 & 26 \\
\hline G3 & 24 & 53 \\
\hline G4 & 2 & 4 \\
\hline \multicolumn{3}{|l|}{ Histological diagnosis } \\
\hline Signet-ring cell carcinoma & 1 & 2 \\
\hline Prior gastrectomy: unknown & 1 & 2 \\
\hline Yes & 24 & 53 \\
\hline No & 21 & 45 \\
\hline Prior adjuvant chemotherapy: unknown & 2 & 4 \\
\hline Yes & 4 & 9 \\
\hline No & 40 & 87 \\
\hline \multicolumn{3}{|l|}{ Disease site } \\
\hline Stomach & 21 & 45 \\
\hline Lymph nodes & 36 & 78 \\
\hline Liver & 22 & 47 \\
\hline Peritoneum & 8 & 17 \\
\hline Bone & 1 & 2 \\
\hline Lung & 3 & 6 \\
\hline
\end{tabular}


3 had a PS of $2(6 \%)$. Most of the patients had histologically confirmed adenocarcinoma $(98 \%)$; the most frequent histological grade was G3 (53\%). Only 2 patients had a gastroesophageal junction cancer. Four patients (9\%) had received prior adjuvant chemotherapy. The most common disease sites were lymph nodes $(78 \%)$, liver (47\%), stomach (45\%), peritoneum (17\%), lung (6\%), and bone $(2 \%)$.

Efficacy and safety

A median of four cycles (range, one to six) per patient was administered. Twenty-eight patients $(61 \%)$ received four cycles of treatment and not six as planned because of toxicity. Moreover, 5 patients received fewer than four cycles (3, early disease progression; 1 , early death from toxicity; 1 , treated with radiotherapy). Twelve patients received six well-tolerated cycles of therapy and all showed a disease response. Twenty-two patients (46\%) were treated at full doses without any delay, thus respecting the dose-dense schedule. The main cause of noncompliance with the dose-dense schedule was hematological toxicity.

Forty-three $(93 \%)$ patients were evaluable for response. Three complete responses (CR) (7\%), 25 partial responses (PR) $(54 \%), 10$ stable disease (SD) $(21 \%)$, and 5 progression of disease (PD) $(11 \%)$ were observed, for an ORR by intention to treat (ITT) analysis of $61 \%(95 \% \mathrm{CI}$, 47-75) (Table 2).

As far as age is concerned ( $\geq 65$ years), 24 patients were evaluable for response. In this population we registered 2 CR (8\%), 14 PR (58 \%), 5 SD (21\%), and 3 PD (13\%); similarly, in younger patients, $1 \mathrm{CR}(5 \%), 11 \mathrm{PR}(58 \%), 5$ SD $(26 \%)$, and 2 PD (11\%) were observed.

After a median follow-up of 23 months $(95 \% \mathrm{CI}$, 13-35), median OS was 17.63 months (95\% CI, 13.67-20.67) and median PFS was 8.9 months (95\% CI, 6.5-13.4) (Figs. 1, 2).

Thirty-eight patients $(83 \%)$ received a second-line therapy after disease progression (37 were treated with chemotherapy and 1 with radiotherapy). Most of the patients received a scheme containing three drugs (oxaliplatin, irinotecan, and 5-FU), in the context of a subsequent clinical trial.

It is noteworthy that two patients initially treated with TCF-dd underwent gastrectomy.

Toxicities observed during treatment are listed in Table 3. All patients were evaluated for toxicity. The most frequent grade 3-4 toxicities were neutropenia (20\%), leukopenia (4\%), thrombocytopenia (2\%), anemia ( $2 \%)$, febrile neutropenia $(6 \%)$, asthenia ( $22 \%$ ), diarrhea (4\%), nausea/vomiting (11\%), and hypokalemia (6\%).
Table 2 Efficacy: intention-to-treat analysis

\begin{tabular}{lrr}
\hline Response & $n$ & $\%$ \\
\hline Complete & 3 & 7 \\
Partial & 25 & 54 \\
Stable disease & 10 & 21 \\
Overall control rate $^{\mathrm{a}}$ & 38 & 82 \\
Progressive disease $_{\text {Not assessable }}$ & 5 & 11 \\
\hline
\end{tabular}

$C R$ complete response, $P R$ partial response, $S D$ stable disease

${ }^{\mathrm{a}} \mathrm{CR}+\mathrm{PR}+\mathrm{SD}$

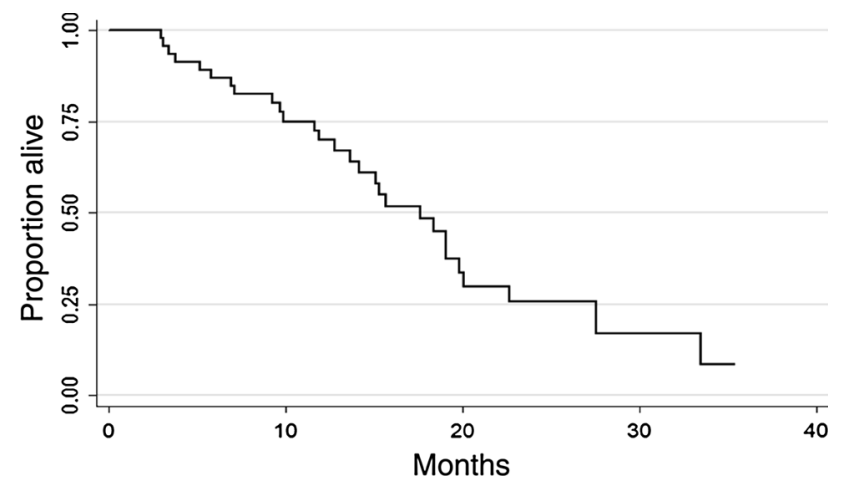

Fig. 1 Kaplan-Meier estimates for overall survival

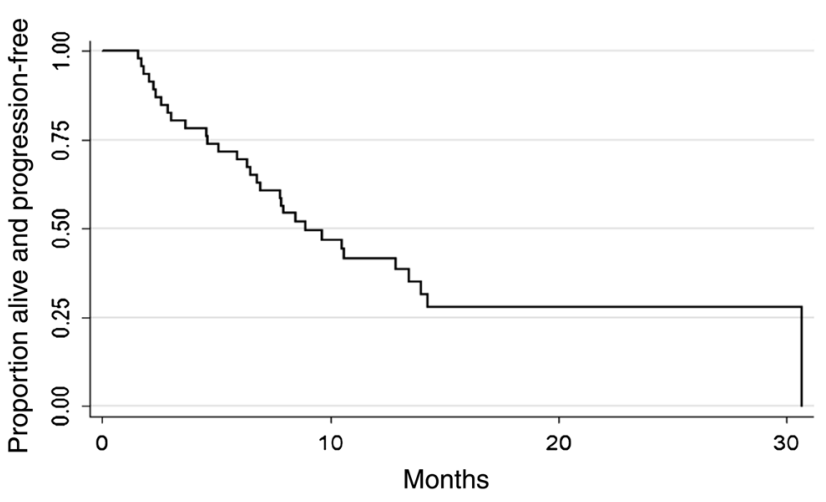

Fig. 2 Kaplan-Meier estimates for progression-free survival

In patients aged $\geq 65$ years, the most frequent grade 3-4 toxicities were neutropenia ( $8 \%$ ), asthenia (16\%), diarrhea $(4 \%)$, nausea/vomiting $(8 \%)$, and hypokalemia (4\%); in younger patients, neutropenia (33\%), asthenia (29\%), diarrhea (5\%), nausea/vomiting (14\%), and hypokalemia $(9 \%)$ were seen. No febrile neutropenia events were encountered in older subjects. 
Table 3 Toxicity according to NCI CTC version 3.0 criteria

\begin{tabular}{|c|c|c|}
\hline & $n=46$ & $\%$ \\
\hline \multicolumn{3}{|l|}{ Leukopenia } \\
\hline Grade $1-2$ & 18 & 39 \\
\hline Grade $3-4$ & 2 & \\
\hline \multicolumn{3}{|l|}{ Neutropenia } \\
\hline Grade $1-2$ & 6 & 13 \\
\hline Grade $3-4$ & 9 & 20 \\
\hline Febrile neutropenia & 3 & 6 \\
\hline \multicolumn{3}{|l|}{ Thrombocytopenia } \\
\hline Grade $1-2$ & 15 & 33 \\
\hline Grade $3-4$ & 1 & 2 \\
\hline \multicolumn{3}{|l|}{ Anemia } \\
\hline Grade $1-2$ & 41 & 89 \\
\hline Grade 3-4 & 1 & 2 \\
\hline \multicolumn{3}{|l|}{ Nausea/vomiting } \\
\hline Grade $1-2$ & 35 & 76 \\
\hline Grade $3-4$ & 5 & 11 \\
\hline \multicolumn{3}{|l|}{ Diarrhea } \\
\hline Grade $1-2$ & 29 & 63 \\
\hline Grade $3-4$ & 2 & 4 \\
\hline \multicolumn{3}{|l|}{ Hypokalemia } \\
\hline Grade $1-2$ & 31 & 67 \\
\hline Grade $3-4$ & 3 & 6 \\
\hline \multicolumn{3}{|l|}{ Asthenia } \\
\hline Grade $1-2$ & 34 & 74 \\
\hline Grade $3-4$ & 10 & 22 \\
\hline Toxic deaths & 1 & 2 \\
\hline
\end{tabular}

\section{Discussion}

The response rate we obtained with TCF-dd $(61 \%)$ is much higher than that reported in previous studies with this combination $[15,16]$.

To increase the percentage of responses it was not necessary to increase drug dosage. In our protocol the following doses were used: docetaxel $70 \mathrm{mg} / \mathrm{m}^{2}$, cisplatin $60 \mathrm{mg} / \mathrm{m}^{2}$, bolus 5 -FU $400 \mathrm{mg} / \mathrm{m}^{2}$, and 5 -FU by continuous infusion for $44 \mathrm{~h}$ at $600 \mathrm{mg} / \mathrm{m}^{2}$. Nearly half the patients $(46 \%)$ complied with the dose-dense criterion without receiving any dose reduction. In the remaining $54 \%$ of patients, it was necessary to reduce the dosage or delay the cycle of chemotherapy because of $\geq \mathrm{G} 2$ hematological or gastrointestinal toxicity.

In the study by Van Cutsem and colleagues [16], docetaxel and cisplatin were administered at higher doses, both $75 \mathrm{mg} / \mathrm{m}^{2}$ on day 1 and 5 -FU at a dose of $750 \mathrm{mg} / \mathrm{m}^{2}$ from day 1 to day 5, every 3 weeks. In $64 \%$ of cases, cycles were postponed, and in $41 \%$ of cases, dose was reduced.
In the Roth et al. study [15], the initial docetaxel dose was $85 \mathrm{mg} / \mathrm{m}^{2}$, but after the first 29 patients enrolled, a protocol amendment was made to reduce the dose to $75 \mathrm{mg} / \mathrm{m}^{2}$ because of the high incidence of febrile neutropenia.

In 2006 the randomized comparison between DCF (docetaxel, cisplatin, and 5-FU) and CF (cisplatin and 5-FU) showed an increase in time to progression (TTP) (5.6 vs. 3.7 months) and OS (9.2 vs. 8.6 months) [16].

In 2007, three different regimens were compared: ECF (epirubicin, cisplatin, and 5-FU) versus TC (docetaxel and cisplatin) versus TCF (docetaxel, cisplatin, and 5-FU), showing a median survival of 8.3, 11.0, and 10.4 months, respectively [15].

In this study, median OS was 17.63 months and TTP 10.67 months. Although these were not the primary endpoints and their value is only descriptive, they deserve to be highlighted.

The median survival in the present study was much longer than in our previous one [19], probably because we administered to all patients lower doses of docetaxel (70 vs. $85 \mathrm{mg} / \mathrm{m}^{2}$ ) and cisplatin $\left(60 \mathrm{vs} .75 \mathrm{mg} / \mathrm{m}^{2}\right.$ ) from the beginning; this modification resulted in a higher response rate (61 vs. $56 \%$ ) combined with lesser hematological toxicity (neutropenia, 20 vs. $53 \%$; febrile neutropenia, 6 vs. $22 \%$ ).

One could correctly argue that this is a phase II study, but efficacy results are among the highest ever reported with this combination.

In all studies containing the triplet docetaxel, cisplatin, and fluorouracil, major treatment-related concerns were represented by hematological and nonhematological toxicity. Taking again into consideration all the limits of this trial (phase II, selected population, lack of control group, etc.), if we make an indirect comparison with the V325 trial [16], we can easily notice that TCF-dd was likely associated with a better tolerability. One of the reasons for these results probably relies on the prophylactic use of pegfilgrastim, which resulted in a better compliance to treatment and allowed us to limit the febrile neutropenia cases to $6 \%$ and G3-G4 neutropenia to $20 \%$, much less than reported in the literature (G3G4 neutropenia $>80 \%$ and febrile neutropenia in $29 \%$ of the patients).

Additionally, G3-G4 gastrointestinal toxicities (diarrhea and nausea/vomiting) were also much lower and well controlled compared with classical TCF (4 vs. $19 \%$ and 11 vs. $14 \%$, respectively).

Among nonhematological side effects, asthenia was particularly frequent and severe in $22 \%$ of patients. We recorded one toxic death from septic shock. Overall, TCFdd was shown to be safe. 
To reduce the significant toxicity related to TCF while maintaining its efficacy, several schedules with different modes of administration have been explored.

In a phase II study, including 60 patients, the administration of docetaxel and cisplatin on days 1, 15, and 29 every 8 weeks with weekly fluorouracil produced an overall response rate of $47 \%$ with a TTP of 8.1 months and an OS of 15.1 months. Rate of febrile neutropenia was $5 \%$ [25].

More recently, an Italian study [26] tested the administration of cisplatin and fluorouracil every 2 weeks for four cycles, sequentially followed by docetaxel every 3 weeks in case of response or stable disease. The aim of the study, which enrolled 34 patients, was to use the three most effective drugs for gastric cancer, but with a modified schedule, to reduce toxicity. Response rate was $38.2 \%$ with PFS and OS of 4.8 and 10.6 months, respectively. The rate of febrile neutropenia reported was $11.8 \%$.

Despite the intensity of our therapeutic scheme, because of the association of three potential toxic drugs and the close interval between cycles, safety apparently was not compromised. This design translated into a relatively high compliance to treatment and into an impressive response rate that has never previously been registered in a phase II study with the same combination.

Possible explanations of these results probably rely on the appropriate selection of the population in study. In effect, the median age was not particularly high (66 years), and $94 \%$ of patients had a PS $=0-1$.

These findings confirm that in specific settings such as good clinical baseline conditions, young age, and low tumoral burden, an intensified chemotherapy regimen such as that used in this protocol may find its proper placing.

Nevertheless, more and more efforts are needed to identify new reliable molecular biomarkers of response to chemotherapy and OS.

Acknowledgments A research grant was received from Amgen.

\section{References}

1. Nagini S. Carcinoma of the stomach: a review of epidemiology, pathogenesis, molecular genetics and chemoprevention. World J Gastrointest Oncol. 2012;4:156-69.

2. Yang D, Hendifar A, Lenz C, et al. Survival of metastatic gastric cancer: significance of age, sex and race/ethnicity. J Gastrointest Oncol. 2011;2:77-84.

3. Glimelius B, Ekstrom K, Hoffman K, et al. Randomized comparison between chemotherapy plus best supportive care with best supportive care in advanced gastric cancer. Ann Oncol. 1997;8:163-8.

4. Murad AM, Santiago FF, Petroianu A, et al. Modified therapy with 5-fluorouracil, doxorubicin, and methotrexate in advanced gastric cancer. Cancer (Phila). 1993;72:37-41.
5. Pyrhonen S, Kuitunen T, Nyandoto P, Kouri M. Randomised comparison of fluorouracil, epidoxorubicin and methotrexate (FEMTX) plus supportive care with supportive care alone in patients with non-resectable gastric cancer. $\mathrm{Br} \mathrm{J}$ Cancer. 1995;71:587-91.

6. Wagner AD, Unverzagt S, Grothe W et al. Chemotherapy for advanced gastric cancer. Cochrane Database Syst Rev 2010; CD004064.

7. Ohtsu A, Shimada Y, Shirao K, et al. Randomized phase III trial of fluorouracil alone versus fluorouracil plus cisplatin versus uracil and tegafur plus mitomycin in patients with unresectable, advanced gastric cancer: the Japan Clinical Oncology Group Study (JCOG9205). J Clin Oncol. 2003;21:54-9.

8. Koizumi W, Narahara H, Hara T, et al. S-1 plus cisplatin versus S-1 alone for first-line treatment of advanced gastric cancer (SPIRITS trial): a phase III trial. Lancet Oncol. 2008;9:215-21.

9. Cullinan SA, Moertel CG, Fleming TR, et al. A comparison of three chemotherapeutic regimens in the treatment of advanced pancreatic and gastric carcinoma. Fluorouracil vs fluorouracil and doxorubicin vs. fluorouracil, doxorubicin, and mitomycin. JAMA. 1985;253:2061-7.

10. Cunningham D, Starling N, Rao S, et al. Capecitabine and oxaliplatin for advanced esophagogastric cancer. $\mathrm{N}$ Engl $\mathrm{J}$ Med. 2008;358:36-46.

11. Webb A, Cunningham D, Scarffe JH, et al. Randomized trial comparing epirubicin, cisplatin, and fluorouracil versus fluorouracil, doxorubicin, and methotrexate in advanced esophagogastric cancer. J Clin Oncol. 1997;15:261-7.

12. Mavroudis D, Kourousis C, Androulakis N, et al. Frontline treatment of advanced gastric cancer with docetaxel and granulocyte colony-stimulating factor (G-CSF): a phase II trial. Am J Clin Oncol. 2000;23:341-4.

13. Ajani JA, Ilson DH, Daugherty K, et al. Activity of taxol in patients with squamous cell carcinoma and adenocarcinoma of the esophagus. J Natl Cancer Inst. 1994;86:1086-91.

14. Kanat O, Evrensel T, Manavoglu O, et al. Single-agent irinotecan as second-line treatment for advanced gastric cancer. Tumori. 2003;89:405-7.

15. Roth AD, Fazio N, Stupp R, et al. Docetaxel, cisplatin, and fluorouracil; docetaxel and cisplatin; and epirubicin, cisplatin, and fluorouracil as systemic treatment for advanced gastric carcinoma: a randomized phase II trial of the Swiss Group for Clinical Cancer Research. J Clin Oncol. 2007;25:3217-23.

16. Van Cutsem E, Moiseyenko VM, Tjulandin S, et al. Phase III study of docetaxel and cisplatin plus fluorouracil compared with cisplatin and fluorouracil as first-line therapy for advanced gastric cancer: a report of the V325 Study Group. J Clin Oncol. 2006;24:4991-7.

17. Ajani JA, Moiseyenko VM, Tjulandin S, et al. Clinical benefit with docetaxel plus fluorouracil and cisplatin compared with cisplatin and fluorouracil in a phase III trial of advanced gastric or gastroesophageal cancer adenocarcinoma: the V-325 Study Group. J Clin Oncol. 2007;25:3205-9.

18. Ajani JA, Moiseyenko VM, Tjulandin S, et al. Quality of life with docetaxel plus cisplatin and fluorouracil compared with cisplatin and fluorouracil from a phase III trial for advanced gastric or gastroesophageal adenocarcinoma: the V-325 Study Group. J Clin Oncol. 2007;25:3210-6.

19. Tomasello G, Chiesa MD, Buti S, et al. Dose-dense chemotherapy in metastatic gastric cancer with a modified docetaxel-cisplatin-5-fluorouracil regimen. Tumori. 2010;96:48-53.

20. Citron ML. Dose-dense chemotherapy: principles, clinical results and future perspectives. Breast Care (Basel). 2008;3:251-5.

21. Held G, Schubert J, Reiser M, Pfreundschuh M. Dose-intensified treatment of advanced-stage diffuse large B-cell lymphomas. Semin Hematol. 2006;43:221-9. 
22. Sternberg CN, de Mulder PH, Schornagel JH, et al. Randomized phase III trial of high-dose-intensity methotrexate, vinblastine, doxorubicin, and cisplatin (MVAC) chemotherapy and recombinant human granulocyte colony-stimulating factor versus classic MVAC in advanced urothelial tract tumors: European Organization for Research and Treatment of Cancer Protocol no. 30924. J Clin Oncol. 2001;19:2638-46.

23. Norton L, Simon R. The Norton-Simon hypothesis revisited. Cancer Treat Rep. 1986;70:163-9.

24. Fizazi K, Zelek L. Is one cycle every three or four weeks obsolete? A critical review of dose-dense chemotherapy in solid neoplasms. Ann Oncol. 2000;11:133-49.
25. Lorenzen S, Hentrich M, Haberl C, et al. Split-dose docetaxel, cisplatin and leucovorin/fluorouracil as first-line therapy in advanced gastric cancer and adenocarcinoma of the gastroesophageal junction: results of a phase II trial. Ann Oncol. 2007;18:1673-9.

26. Catalano V, Vincenzi B, Giordani P, et al. Sequential chemotherapy with cisplatin, leucovorin, and 5-fluorouracil followed by docetaxel in previously untreated patients with metastatic gastric cancer: a phase II study. Gastric Cancer. 2012;15:419-26. 